\title{
Erratum to: Attractor Dynamics and Thermodynamic Analogies in the Cerebral Cortex: Synchronous Oscillation, the Background EEG, and the Regulation of Attention
}

\section{J.J. Wright}

Published online: 10 February 2011

(C) Society for Mathematical Biology 2011

\section{Erratum to: Bull Math Biol \\ DOI 10.1007/s11538-010-9562-z}

The reference Potthast, R. P., beim Graben, P., \& Wright, J. J. (2010) should have read as follows:

Potthast, R. P., beim Graben, P., \& Wright, J. J. (in prep). Emergence of cortical maps through synaptic competition and cooperation dynamics.

The online version of the original article can be found under doi:10.1007/s11538-010-9562-z.

J.J. Wright (凶)

Liggins Institute and Department of Psychological Medicine, University of Auckland, Auckland, New Zealand

e-mail: jj.w@xtra.co.nz 